3th ICFA ilivanced Beam Dynamic Workshop on Space Charge Dominated Beams and $\Gamma A m p l i c a t i o n s$ of Higil brightness Beams, Bloomington, IN, 10/11-13/95.

\title{
Reducing space charge tune shift with a barrier cavity ${ }^{1}$
}

M. Blaskiewicz

AGS Dept. Brookhaven National Lab

\footnotetext{
Implementation of a barrier cavity if system appears to be a straightforward and relatively inexpensive way to increase the output current of second stage synchrotrons. This note serves as a general introduction to the relevant beam dynamies and addresses the problem of driving a cavity in the short burst mode.
}

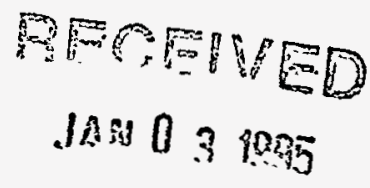

\section{INTRODUCTION}

A barrier cavity if system $(1,2)$ is proposed as a tool in the continuing effort to obtain higher beam currents. Unlike a single frequency if system a barrier cavity if system employs a single turn voltage waveform similar to that shown in Figure 1. Each of the pulses making up the waveform tends to repel particles resulting in two bunches separated by narrow gaps. By varying the amplitude and phase of the pulses some novel if manipulations become possible.

\section{SINGLE PARTICLE BEAM DYNAMICS}

Before proceeding it will be useful to define some relevant coordinates. The azimuth $\theta$ refers to longitudinal position with respect to the physical machine and increases by $2 \pi$ each turn. I assume the RF field is limited to a narrow range around $\theta=0$, several gaps will be similar as long as the time delays are correct. The RF system creates an electric field whose component along the beam velocity is given by $V(t) \delta_{p}(\theta) / R$ where $V(t)$ is the gap voltage as a function of time, $R$ is the machine radius and $\delta_{p}(\theta)$ is the periodic delta function. I assume a constant magnetic field and an ideal energy $E_{0}$ with corresponding angular frequency $\omega_{0}$. A comoving coordinate is defined by the relative phase $\phi=\theta-\omega_{0} t$. Let $\phi_{n}$ and $E_{n}$ correspond to the relative phase and the total energy a particle has just after traversing the cavity for the $n$th time. To leading order and neglecting collective effects,

$$
\phi_{n+1}-\phi_{n}=-\frac{2 \pi \eta\left(E_{n}-E_{0}\right)}{\beta^{2} E_{0}}
$$

\footnotetext{
${ }^{1}$ Work performed under the auspices of the U.S. Department of Energy.
} 


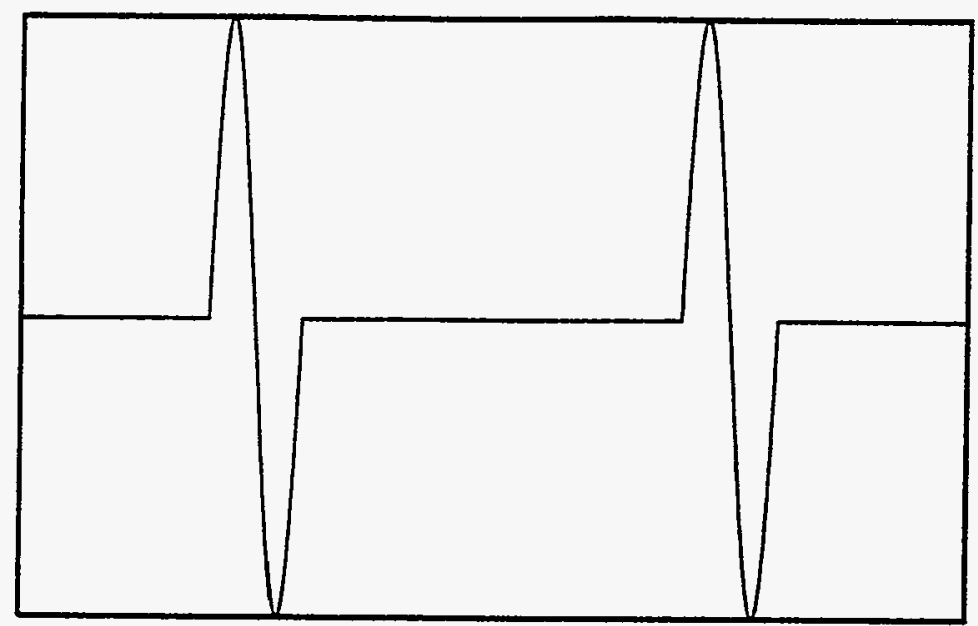

FIG. 1. Voltage .7s. time for one revolntion period. The pheses and amplitudes of the two components may vary slowly from one turn to the next.

$$
E_{n+1}-E_{n}=q V\left(n T_{0}-\phi_{n+1} / \omega_{0}\right)
$$

where $q$ is the charge of the particle, $\eta$ is the frequency slip factor, $\beta=v / c$ and $T_{0}$ is the revolution period. The difference equations can be approximated by differential equations. With $n$ as the smooth time-like variable the differential equations for coordinate $\phi$ with conjugate momentum $E$ can be obtained from the Hamiltonian,

$$
H(\phi, E)=-\frac{\pi \eta\left(E-E_{0}\right)^{2}}{\beta^{2} E_{0}}+U(\phi) .
$$

The potential is given by

$$
U(\phi)=-q \int_{0}^{\phi} V\left(x / \omega_{0}\right) d x
$$

Slow changes in the confining potential can be included in the usual way to produce $U(t, \phi)$. As long as $U(t, \phi)$ varies slowly enough in $t$, the adiabatic invariant given by $J=\oint E d \phi$, where the integral is over one period of the motion, will be nearly conserved. Figure 2 shows simulation results for a possible scenario using the AGS and AGS Booster which have a $4: 1$ circumference ratio. To make the connection with adiabatic invariants visible $\sqrt{H}$ and $\sqrt{U}$ are plotted. Figure $2 \mathrm{a}$ shows four Booster cycles worth of particles 


\section{DISCLAIMER}

Portions of this document may be illegible in electronic image products. Images are produced from the best available original document. 
outside the barrier bucket and another Booster cycie has just been injected. In Figure $2 b$ the injected particles have been squeezed yielding a similar energy distribution for particles inside and outside the bucket. In Figure $2 c$ the second voltage component has collapsed. In $2 \mathrm{~d}$ this component has returned in phase with the first. In Figures $2 e$ and $f$ the voltage components separate and grow making room for the next Booster cycle. In plot units, the initial peak momentum is 4 while the final peak momentum is $\mathbf{5}$ and the number of particles outside the barrier increases in the same ratio. There is very little emittance dilution during the process.

\section{MULTIPARTICLE BEAM DYNAMICS}

From a multiparticle standpoint a barrier cavity if system has several features which distinguish it from a single frequency if system. By optimizing the injection process it is possible to reduce the peak current without significantly diluting the longitudinal emittance. Additionally, the peak current in a barrier cavity system is time dependent, growing with each transfer. Reducing the peak current results in a smaller space charge tune shift which should reduce stopband losses. As an example consider a single bunch in the Booster having a parabolic line density of full length equal to half the Booster circumference. The gap in the AGS (cf. Figure 2) is taken to be the same length. Figure 3 shows the peak current in the AGS in units of Booster peak current assuming that the initial filamentation of the parabolic bunch in the gap is essentially instantaneous. The initial filamentation causes a fractional emittance increase of $4 / \pi$ and reduces the peak current to $2 / 3$ of the Booster value. Notice that the peak current for the stored bunch does not reach the initial injected intensity until the sixth Booster transfer and that the peak current for eight transfers always remains below the peak current in the Booster. After the injection process is completed the beam could be captured in a conventional if system and quickly accelerated. The important point is that the time average space charge tune depression is greatly reduced with the barrier cavity system.

Coherent instabilities with a barrier cavity of system are different from the sort found with a single frequency if system. When the barrier half width is small compared to the bunch length $\left(\phi_{b}\right)$, the synchrotron tune $\left(\nu_{s}\right)$ is proportional to the fractional energy deviation,

$$
\nu_{\triangleleft} \approx \frac{\pi}{\phi_{b} \beta^{2}}\left|\eta \frac{E-E_{0}}{E_{0}}\right| .
$$

The large spread in synchrotron tune implies a lot of Landau damping and in a first approximation the coasting beam stability criteria with the same peak current should give reasonable limits. 

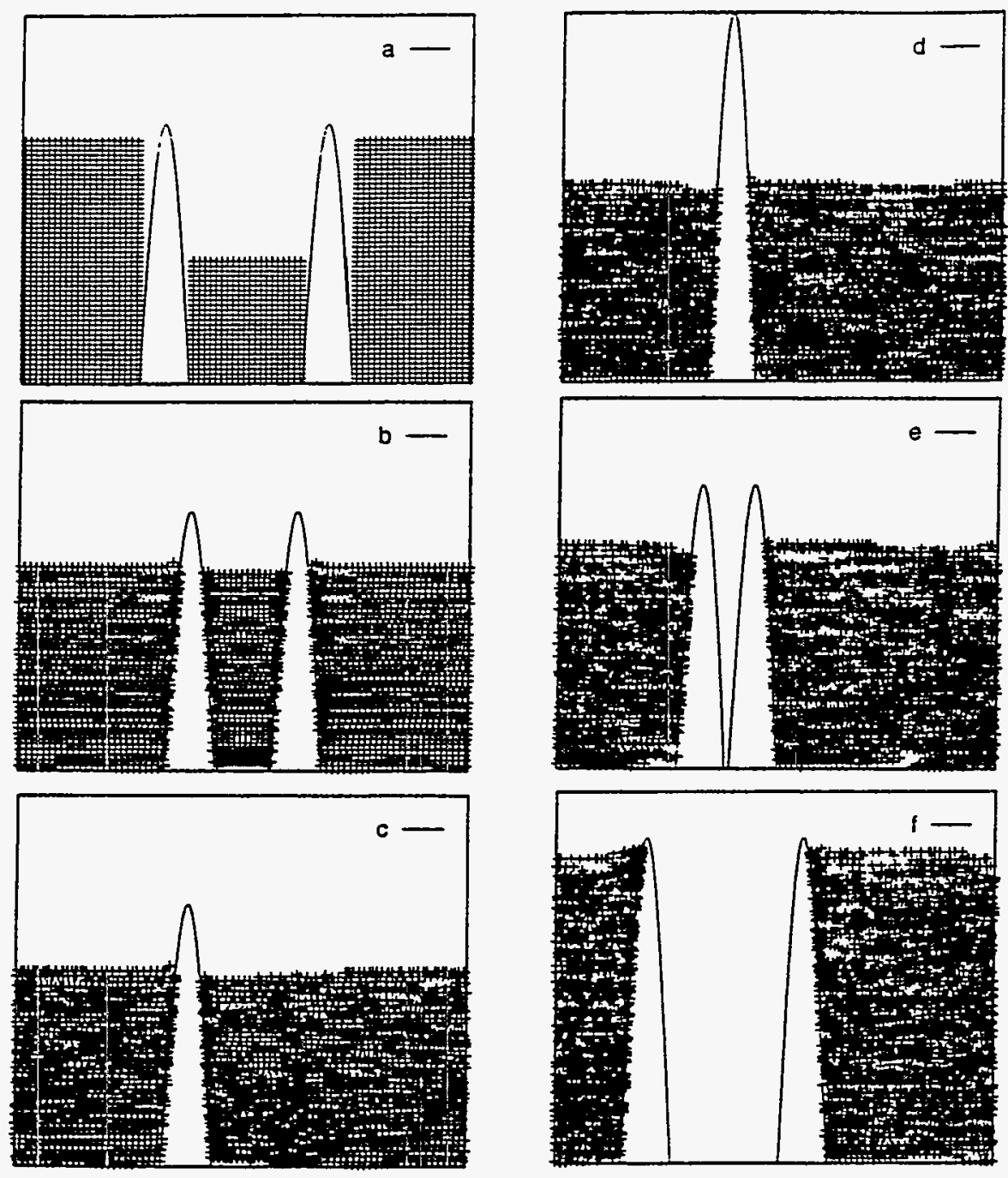

FIG. 2. Simulation of an injection scenario using a barrier cavity sf system. 


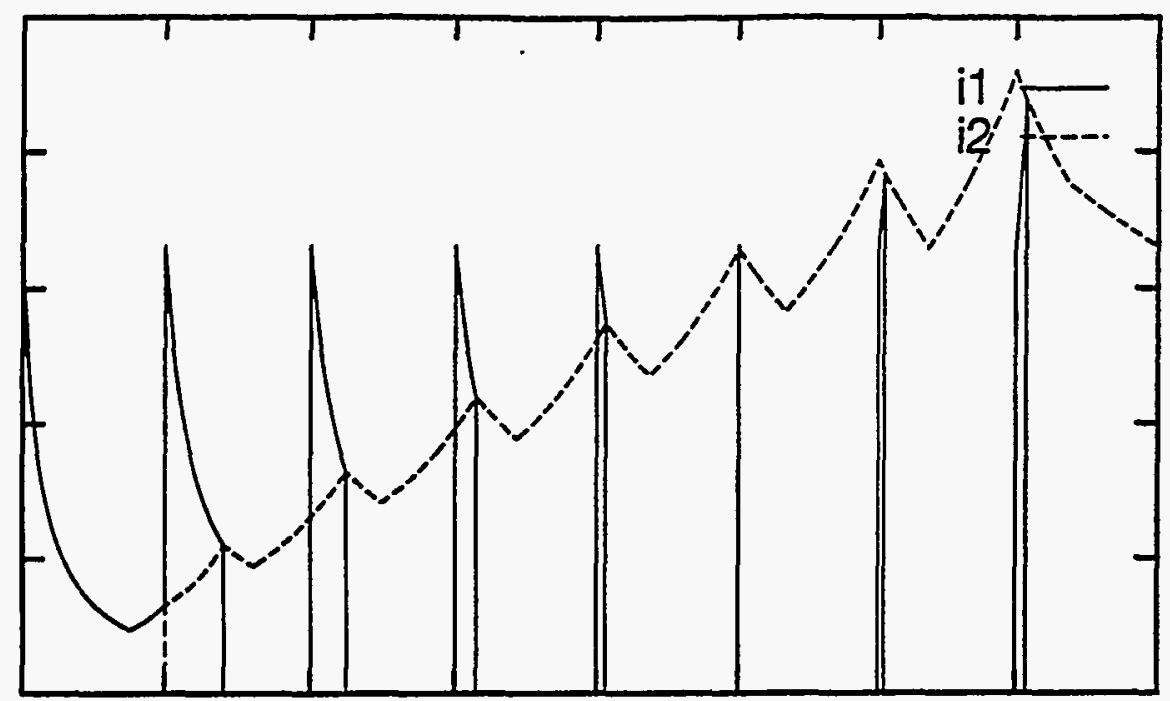

FIG. 3. (Peak current in AGS)/(Peak current in Booster) for eight Booster transfers.

\section{RF CONSIDERATIONS}

Generating an if waveform like that shown in Figure 1 can be done in at least two ways. The first way is to approximate the waveform by a truncated Fourier series and to create the net voltage per turn using several single frequency if systems operating at several revolution frequency harmonics. This appears to be a fairly complicated solution and will not be considered further.

Another way to generate the voltage waveform in Figure 1 is to asaume that only a single type of of cavity is available and to tailor the current pulse driving the cavity to create the desired voltage. For simplicity assume that the cavity impedance can be characterized by a single resonator

$$
Z(\omega)=\frac{R_{s}}{1+i Q\left(\omega_{r} / \omega-\omega / \omega_{r}\right)}
$$

where $R_{d}$ is the shunt impedance, $Q$ is the quality factor, and $\omega_{r}$ is the resonant frequency. For a current pulse $I(t)$ the voltage across the cavity is given by

$$
\begin{aligned}
& V(t)=\int_{0}^{\infty} I(t-\tau) W(\tau) d \tau \\
& W(t)=\frac{\omega_{r} R_{t}}{\tilde{\omega} Q} \frac{d}{d t} \sin (\bar{\omega} t) e^{-\omega_{r} t / 2 Q}
\end{aligned}
$$


where $W(t)$ is the wake potential and $\bar{\omega}=\omega_{r} \sqrt{1-1 / 4 Q^{2}}$. The cavity need not ring after the current pulse is turned off. Let $I_{0}(t)$ be some easily created current pulse of finite duration. Driving the cavity with a current $I(t)=$ $I_{0}(t)+\exp \left[-\pi / \sqrt{\left(4 Q^{2}-1\right)}\right] I_{0}(t-\pi / \bar{\omega})$ results in no cavity voltage after the current $I(t)$ stops flowing. Another way to create a voltage pulse of finite duration is to define the voltage pulse required and then, via Fourier analysis, calculate the necessary current pulse. A particularly simple result is (4)

$$
\begin{gathered}
I(t)= \begin{cases}I_{0}\left(1+\frac{\sin \left(\omega_{r} t\right)}{Q}\right), & 0<t<2 \pi / \omega_{r} \\
0, & \text { otherwise }\end{cases} \\
V(t)= \begin{cases}\frac{I_{0} R_{s}}{Q} \sin \left(\omega_{r} t\right), & 0<t<2 \pi / \omega_{r} \\
0, & \text { otherwise. }\end{cases}
\end{gathered}
$$

Note that the discontinuity of the current in equation 9 is not a fundamental problem. Convolving the current pulse with a Bartlett window (isosceles triangle function) yields a current pulse that is continuous with continuous derivative and smooths out the voltage without creating any ringing.

The results of a hardware test are shown in Figure 4 (3). The persistent high frequency ringing after the main voltage pulse is due to a parasitic resonance and would be removed by active feedback in an operational mode.

\section{CONCLUSIONS}

A barrier cavity if system appears feasible on both theoretical and practical grounds. The main effect of such a system is to reduce the time average of the peak current. The benefits involve a reduction in space charge tune shift and an increase in synchrotron frequency spread. Longitudinal phase space dilution is small. Since the if system generally contributes a small fraction to the total cost of a synchrotron the installation of barrier buckets is relatively inexpensive as well.

\section{REFERENCES}

1. J.E. Griffin, C. Ankenbrandt, J.A. MacLachlan, A. Moretti, IEEE TNS, 30, p. 3502, 1983.

2. V.K. Bharadwaj, J.E. Griffin, D.J. Harding, J.A. MacLachlan, IEEE TNS, 34, p. $1025,1987$.

3. J.M. Brennan, private communication.

4. M. Meth, private communication. 


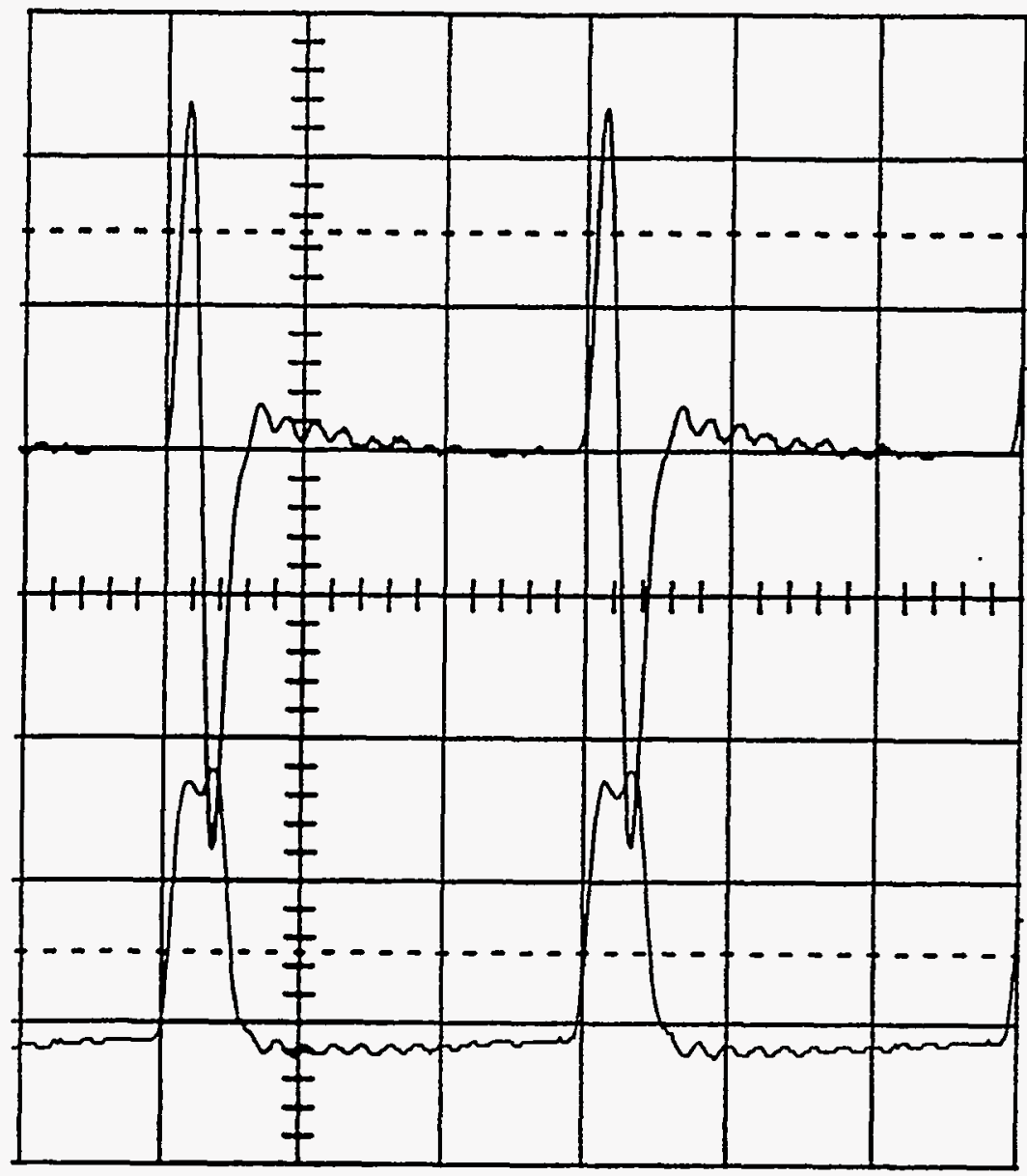

FIG. 4. Experimentel confirmstion of pulsed operation using existing hardware at the AGS. The time increment is $1 \mu \mathrm{s}$ per box. The top trace is the gap voltage (1160 volts per box), while the bottom trace is the trbe current ( 40 amps per box).

\section{DISCLAIMER}

This report was prepared as an account of work sponsored by an agency of the United States Government. Neither the United States Government nor any agency thereof, nor any of their employees, makes any warranty, express or implied, or assumes any legal liability or responsibility for the accuracy, completeness, or usefulness of any information, apparatus, product, or process disclosed, or represents that its use would not infringe privately owned rights. Reference herein to any specific commercial product, process, or service by trade name, trademark, manufacturer, or otherwise does not necessarily constitute or imply its endorsement, recommendation, or favoring by the United States Government or any agency thereof. The views and opinions of authors expressed herein do not necessarily state or reflect those of the United States Government or any agency thereof. 